\title{
ISOLATION OF DIHYDROMYRICETIN AND DIHYDROQUERCETIN FROM BARK OF PINUS CONTORTA
}

\author{
by \\ HENRIK OUTTRUP, KJELD SCHAUMBURG ${ }^{11}$ \\ and \\ JØRGEN ØGAARD MADSEN ${ }^{2\}}$ \\ Department of Brewing Chemistry, Carlsberg Research Center, \\ Gamle Carlsberg Vej 10, DK-2500 Copenhagen Valby \\ "Department of Chemical Physics, The H.C. Ørsted Institute, \\ University of Copenhagen, Universitetsparken 5, DK-2 100 Copenhagen $\varnothing$ \\ ${ }^{21}$ Department of Organic Chemistry, The Technical University of Denmark, \\ DK-2800 Lyngby
}

Keywords: Pinus contorta Loud., shore pine, (+)-dihydromyricetin, (+)-dihydroquercetin, ${ }^{1} \mathrm{H}-{ }^{13} \mathrm{C}$ NMR, mass spectrometry, large-scale extraction

\begin{abstract}
Bark of shore pine has high contents of the dihydroflavonols dihydromyricetin and dihydroquercetin. A method for isolation of the pure, optically active compounds in gram amounts has been developed, involving Soxhlet extraction of finely ground, dry bark and preparative scale reversed-phase HPLC. Among a number of inexpensive solvents tested ethyl acetate is useful, being reasonably effective in the extraction step and giving a crude extractive that is suitable for HPLC without any pre-treatment. The use of bark from dead trees, which have been lying in the open for a couple of years, is advantageous because of the lower content of resinous material. $270 \mathrm{MHz}{ }^{\prime} \mathrm{H}$ - and ${ }^{13} \mathrm{C}-\mathrm{NMR}$ and electron impact mass spectra are presented.
\end{abstract}

\section{INTRODUCTION}

The present work was undertaken in order to obtain substantial quantities of the dihydroflavonols dihydroquercetin (DiHQ), I and, especially dihydromyricetin (DiHM), II. These compounds are needed for the syntheses of specific proanthocyanidins present in barley. The low content $(0.15-0.20 \%)$ and the complexity of the proanthocyanidin mixture of barley $(11,14,15)$, comprising procyanidins, prodelphinidins and mixed proanthocyanidins, make chemical synthesis attractive in order to obtain sufficient material to investigate the effect of individual proanthocyanidins on beer haze formation. All of the barley proanthocyanidins identified so far contain dextrorotatory moities only and have a $(+)$-catechin unit as the flavan-3-ol terminal (11, $14,15)$. Naturally occurring procyanidins have been synthesized from DiHQ $((+)$-taxifolin) and $(+)$-catechin $(4,5,6)$, whereas syntheses of pro-

Abbreviations: $\mathrm{DiHM}=$ dihydromyricetin; $\mathrm{DiHQ}=$ dihydroquercetin; $\mathrm{HPLC}=$ high performance liquid chromatography; MS = mass spectrometry; NMR = nuclear magnetic resonance; TMS = tetramethylsilane. 
delphinidins have not been reported, probably due to lack of the appropriate starting material, DiHM.

DiHM is, compared to DiHQ, a rather seldom encountered compound. It was first isolated in an optically inactive form, named ampelopsin (CA 27200-12-0), from the Chinese drug Ampelopsis meliaefolia Kudo (12). Afterwards dextrorotatory DiHM has been obtained from a number of plant sources, some of which are dealt with in refs. 1, 10, 13. Recently, the first dihydromyricetin glycoside (the 3-O-rhamnoside) was reported (7).

Since dihydroflavonols having positive rotation are considered to possess $2 \mathrm{R}: 3 \mathrm{R}$ ( $2 \mathrm{R}$-trans) stereochemistry (3) the structure of DiHM is most likely represented by formula II.

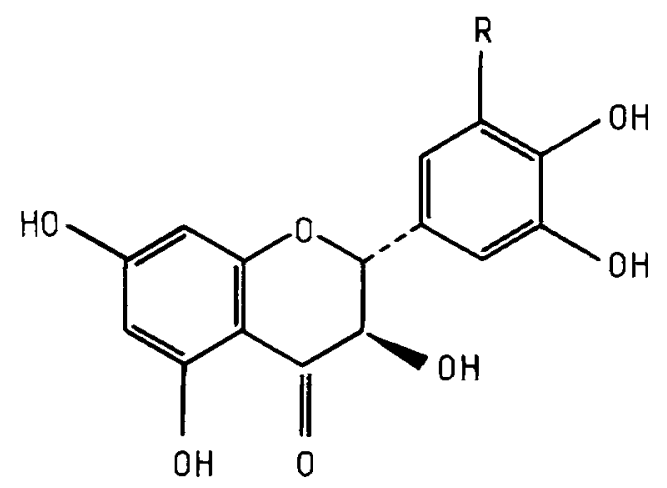

I: $\mathrm{R}=\mathrm{H}$, DiHQ

2,3-dihydro-3,5,7-trihydroxy-

2-(3,4-dihydroxyphenyl)-4H-1-

benzopyran-4-one-(2R-trans).

II: $\mathrm{R}=\mathrm{OH}$, DiHM

2,3-dihydro-3,5,7-trihydroxy-

2-(3,4,5-trihydroxyphenyl)-4H-1-

benzopyran-4-one-(2R-trans).

In our search for a readily accessible source of DiHM we noted that several pine species were reported to be rich in flavonoids (8), and that HERGERT (9) by a large-scale hot water extraction of the bark of the coastal variety of lodgepole pine (shore pine, Pinus contorta Loud. (Dougl.)) obtained a yield of $0.15 \%$ of a 3 to 1 mixture of DiHM and DiHQ. The two compounds could not be separated. The mentioned pine species, endemic to North America, has been introduced into Denmark from the coastal regions of the state of Washington, USA. We have investigated the extraction of the dihydroflavonols from bark of dead and living trees of shore pine with various solvents. Crude extracts from bark of dead trees were subjected to preparative HPLC from which eluate fractions were collected, which after concentration afforded the individual, pure compounds by spontaneous crystallization. Purity and steric integrity were assessed by HPLC, NMR, MS and polarimetry. Our bark materials showed considerably higher contents of the dihydroflavonols than that previously examined (9). We also present results of ' $\mathrm{H}$ - and ${ }^{13} \mathrm{C}-\mathrm{NMR}$ and mass spectrometric analyses, complementing the data in the literature.

\section{MATERIALS AND METHODS \\ 2.1. Bark samples}

Bark was obtained from trees of shore pine grown in North Jutland. The samples were collected in the years 1982 (Sample 1, dead tree, 35 years old), 1983 (Sample 2, dead tree, 52 years old), 1984 (Sample 3, dead tree, 37 years old) and 1985 (Sample 4, living tree, 38 years old).

Samples 1, 3 and 4 were from a plantation (Fosdal) of somewhat uncertain provenience. but of pronounced coastal character. Sample 2 was from an older plantation (Langdal) of certain, coastal provenience. The dead trees had been lying for about two years before the bark was peeled off.

The bark flakes were air-dried at ambient temperature in a drying cupboard for $24 \mathrm{~h}$ and broken into small pieces. Sample 1 was ground directly in a Fuchs Mill, type MM 125 H. Sample 2 was initially ground in a United Milling System type 400 disc mill before final grinding in the Fuchs Mill. Sample 3 was ground in the United Milling System Mill only, whereas sample 4 was ground in the Fuchs Mill only.

\subsection{Solvents}

All organic solvents were of analytical grade (Merck), and used without further purification. The water was deionized and all glass distilled. For HPLC purposes the solvents were appropriately filtered before use. 


\subsection{Extraction}

\subsubsection{Extraction, small-scale procedure}

Bark flour, 4-5 g, was extracted in a Soxhlettype apparatus with $150 \mathrm{ml}$ of solvent for $6 \mathrm{~h}$. The solvent was evaporated in vacuum. Residues of extracts from bark of dead trees (Samples 1-3) were taken up in $10 \mathrm{ml}$ of $50 \%$ aqueous methanol. After centrifugation at $2700 \mathrm{~g}$ for 10 min the supernatant was diluted before HPLC analysis. The residue from Sample 4 was taken up in methanol, centrifuged and diluted with an equal amount of water. This solution had to be centrifuged before HPLC analysis.

\subsubsection{Extraction, large-scale procedure}

Bark flour, $600 \mathrm{~g}$, was packed in a cartridge made of Whatman No. 1 filter paper and extracted for $6 \mathrm{~h}$ in a 21 Soxhlet-type apparatus equipped with a $5 \mathrm{l}$ reservoir loaded with $2.5 \mathrm{l}$ of solvent. The extract was kept overnight at $5^{\circ} \mathrm{C}$, whereafter the precipitate was filtered off and the solvent removed on a rotary evaporator at a pressure not lower than 60 Torr in order to prevent vigorous foaming. The solid, crude extract was kept at $-30{ }^{\circ} \mathrm{C}$ until further processing.

\subsection{HPLC}

\subsubsection{Analytical procedure}

Analytical HPLC was performed on a Hewlett-Packard model $1084 \mathrm{~B}$ equipped with a 2.5 $\mathrm{cm} \times 4 \mathrm{~mm}$ (i.d.) and a $12.5 \mathrm{~cm} \times 4 \mathrm{~mm}$ (i.d.) Lichro Cart $7 \mu \mathrm{m}$ C18 column (Merck) in series and a variable wavelength UV-detector, operating at $290 \mathrm{~nm}$. The mobile phases were A: $1.5 \%$ acetic acid in water, and B: $10 \%$ acetic acid in methanol, heated to $70^{\circ} \mathrm{C}$ and $50^{\circ} \mathrm{C}$, respectively. The column temperature was $40^{\circ} \mathrm{C}$. A gradient of 20 to $50 \%$ of solvent $\mathrm{B}$ in solvent $\mathrm{A}$ in $30 \mathrm{~min}$ at a flow rate of $1.0 \mathrm{ml} / \mathrm{min}$ was used. Not more than $10 \mu \mathrm{g}$ of each of the dihydroflavonols was injected. Retention times appear from Figure 1.

\subsubsection{Preparative procedure}

Preparative HPLC was carried out using a Waters Prep LC/system 500A equipped with two Waters Prep Pak/C18 columns in series.
The dihydroflavonols were chromatographed isocratically with water-methanol-acetic acid (68.5:30:1.5) at a flow rate of $150 \mathrm{ml} / \mathrm{min}$ at ambient temperature. The elution was monitored with a refractive index detector. The sample was a centrifuged solution of crude extract containing approx. $6 \mathrm{~g}$ of dihydroflavonols in $250 \mathrm{ml}$ of the mobile phase. The columns were washed thoroughly with $80 \%$ aqueous methanol between runs.

\subsection{NMR spectroscopy}

The ${ }^{1} \mathrm{H}$ spectra were obtained using a Bruker HX 270D spectrometer in Fourier transform mode. Samples were prepared from $10 \mathrm{mg}$ of the appropriate substance dissolved in $300 \mu \mathrm{l}$ of solvent (see Tables II-IV). Tetramethylsilane (TMS) was added as an internal reference. The solutions were placed in $5 \mathrm{~mm}$ o.d. sample tubes. Spectra were accumulated at $293 \mathrm{~K}$ using a spectral width of $3000 \mathrm{~Hz}$ and $32 \mathrm{~K}$ data points.

${ }^{13} \mathrm{C}$ spectra were obtained at $67.889 \mathrm{MHz}$ using the same instrument. Samples were prepared from $100 \mathrm{mg}$ of the substances dissolved in $2 \mathrm{ml}$ of solvent. Chemical shift values are reported using acetone or TMS as internal standard. The samples were placed in $10 \mathrm{~mm}$ o.d. sample tubes. Spectra were accumulated at 300 $\mathrm{K}$ using a spectral width of $17 \mathrm{kHz}$ and $32 \mathrm{~K}$ data points. Pulse width was selected as $8 \mu$ s corresponding to a $50^{\circ}$ flip angle.

The dihydroflavonols were analysed both as the genuine compounds and as the perdeuteroacetyl derivatives prepared as described earlier (14).

\subsection{Mass spectrometry}

Electron impact mass spectra were recorded on a VG 7070F instrument equipped with a VG 2035 Data System. The ionization energy was 70 $\mathrm{eV}$. The ion source temperature was $200^{\circ} \mathrm{C}$.

\subsection{Polarimetry}

Mesurements were performed using a PerkinElmer model 241 polarimeter equipped with a 1 $\mathrm{ml}, 1 \mathrm{dm}$ pathlength cuvette and a circulating water thermostat. 
H. OUtTRup et al.: Dihydromyricetin and dihydroquercetin

Table I Small scale Soxhlet extraction of dry bark.

\begin{tabular}{|c|c|c|c|c|c|}
\hline \multicolumn{2}{|c|}{$\begin{array}{c}\text { Bark } \\
\text { sample }\end{array}$} & \multirow[t]{2}{*}{ Solvent } & \multicolumn{2}{|c|}{$\begin{array}{l}\text { Dihydroflavonols } \\
\mathrm{mg} / \mathrm{g} \text { dry bark }\end{array}$} & \multirow[t]{2}{*}{ DiHM/DiHQ } \\
\hline No. & Condition & & DiHM & DiHQ & \\
\hline \multirow[t]{4}{*}{1} & \multirow[t]{4}{*}{ dead } & ethanol & 3.8 & 6.1 & 0.6 \\
\hline & & ethanol & 3.8 & 2.9 & 1.3 \\
\hline & & methanol & 3.8 & 2.9 & 1.3 \\
\hline & & dioxane & 3.9 & 2.5 & 1.6 \\
\hline \multirow[t]{5}{*}{2} & \multirow[t]{5}{*}{ dead } & water & 3.2 & 1.3 & 2.5 \\
\hline & & ethyl acetate & 3.4 & 2.4 & 1.4 \\
\hline & & ethyl ether & 3.0 & 1.6 & 1.9 \\
\hline & & chloroform & 0.6 & 0.4 & 1.5 \\
\hline & & toluene & $\sim 0$ & $\sim 0$ & - \\
\hline \multirow[t]{2}{*}{3} & \multirow[t]{2}{*}{ dead } & ethanol & 11.4 & 8.0 & 1.4 \\
\hline & & ethyl acetate & 10.6 & 7.1 & 1.5 \\
\hline \multirow[t]{2}{*}{4} & \multirow[t]{2}{*}{ fresh } & ethanol & 4.0 & 3.1 & 1.3 \\
\hline & & ethyl acetate & 2.2 & 1.6 & 1.4 \\
\hline
\end{tabular}

\section{RESULTS AND DISCUSSION}

\subsection{Isolation of dihydroflavonols}

At the initial stage of this work the aim was to isolate the flavonols from shore pine bark, especially myricetin, which upon reduction could be transformed to a dihydromyricetin material that, though being racemic, might be used in the syntheses mentioned in the introduction. HERGERT obtained myricetin from the bark of shore pine in about $2 \%$ yield (9).

It was observed, however, that an ethyl ether extract of bark contained substantial amounts of DiHM and DiHQ, easily recovered from the ethyl ether extract residue with hot water $\left(70^{\circ} \mathrm{C}\right)$. Thus, about $5 \mathrm{~kg}$ of bark (Sample 1) yielded 7.2 $\mathrm{g}$ of DiHM and $4.5 \mathrm{~g}$ of DiHQ. It was therefore decided to search for an efficient single step extraction method and if possible avoiding use of highly inflammable, toxic or expensive solvents.

A number of solvents were tested in small scale Soxhlet extractions (2.3.1) using Sample 2 material. The results are shown in Table $I$. As can be seen the methanol and ethanol are the most efficient. Although the dihydroflavonols are very soluble in hot water this solvent is less efficient especially with respect to extraction of DiHQ. Ethyl ether also shows much reduced efficiency in the extraction of DiHQ. This ex- plains the low yield of DiHQ obtained from bark Sample 1. Solvents like toluene and chloroform are useless.

Thus, when only considering the extraction step of the procedure the solvent of choice would be ethanol. This solvent, however, also dissolves large amounts of polar material, as indicated by the very elevated baseline in the analytical HPLC chromatogram of an ethanol extract, Figure 1a. In preparative HPLC (2.4.2), using refractive index monitoring, these impurities completely mask the dihydroflavonol peaks preventing accurate cutting of fractions. The eluate from the first part of the elution is dark, as is the ethanol extract itself.

Ethyl acetate was then examined as solvent for large-scale extraction. As shown in Figure $1 \mathrm{~b}$ the extract, which is only slightly coloured, appears to be free from early eluting polar material, and preparative HPLC could be carried through easily. Though it is not fully efficient as an extraction solvent (Table I) ethyl acetate, then, seems to be a suitable compromise. Extract residues from $2400 \mathrm{~g}$ of bark (Sample 2) suspended in $500 \mathrm{ml}$ of the mobile phase was centrifuged and loaded in two consecutive runs. A chromatogram (2.4.2) is shown in Figure 2. As can be seen DiHM and DiHQ are completely separated. Minor components appear at the 
H. OUTtRup et al.: Dihydromyricetin and dihydroquercetin
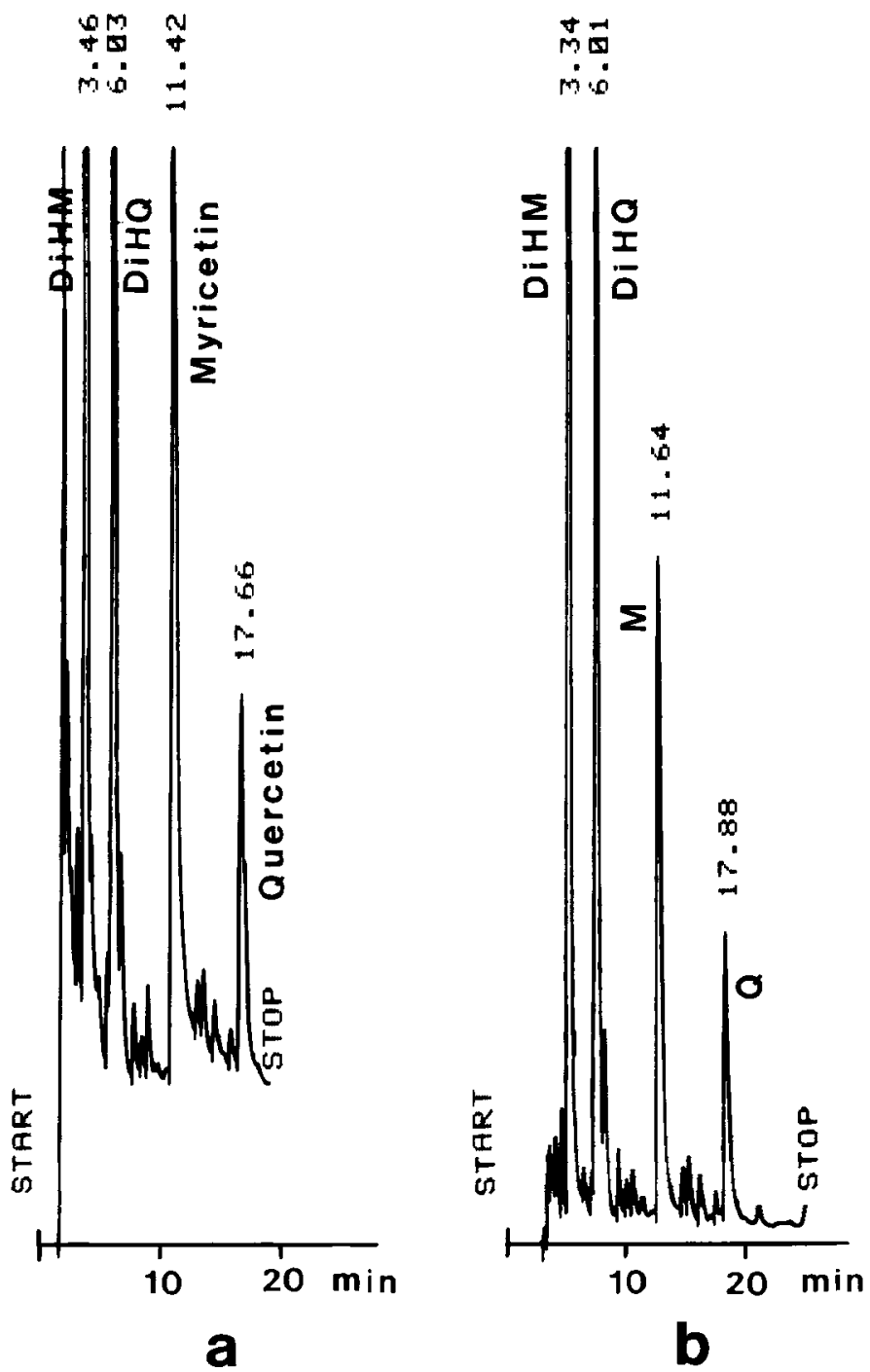

Figure 1. Analytical HPLC chromatograms (2.4.1) of extracts of bark. a: ethanol extract; b: ethyl acetate extract. The ordinates represents 0.256 AUFS.

beginning of the elution close to DiHM. These compounds, however, seem not to coprecipitate with DiHM (see below). The flavonols are not eluted until $80 \%$ aqueous methanol is applied as mobile phase. Fractions containing separately DiHM and DiHQ were concentrated on a rotary evaporator until precipitation of dihydroflavonols had started. After cooling to $5^{\circ} \mathrm{C}$ the compounds were collected by filtration. According to quantitative analytical HPCL of the extract the attainable amounts of DiHM and
DiHQ would be 8.2 and $5.8 \mathrm{~g}$, respectively. The actual yields were $7.6 \mathrm{~g}$ of DiHM and $3.0 \mathrm{~g}$ of DiHQ, i.e. a substantially lower yield than expected of DiHQ. Apparently DiHQ crystallizes less readily than DiHM from the concentrated eluates.

Table I also includes analyses of the other bark samples. There are considerable differences between the samples with respect to both total and relative amounts of dihydroflavonols. Since only one sample of fresh bark from a living tree 
H OUtrRup et al.: Dihydromyricetin and dihydroquercetin

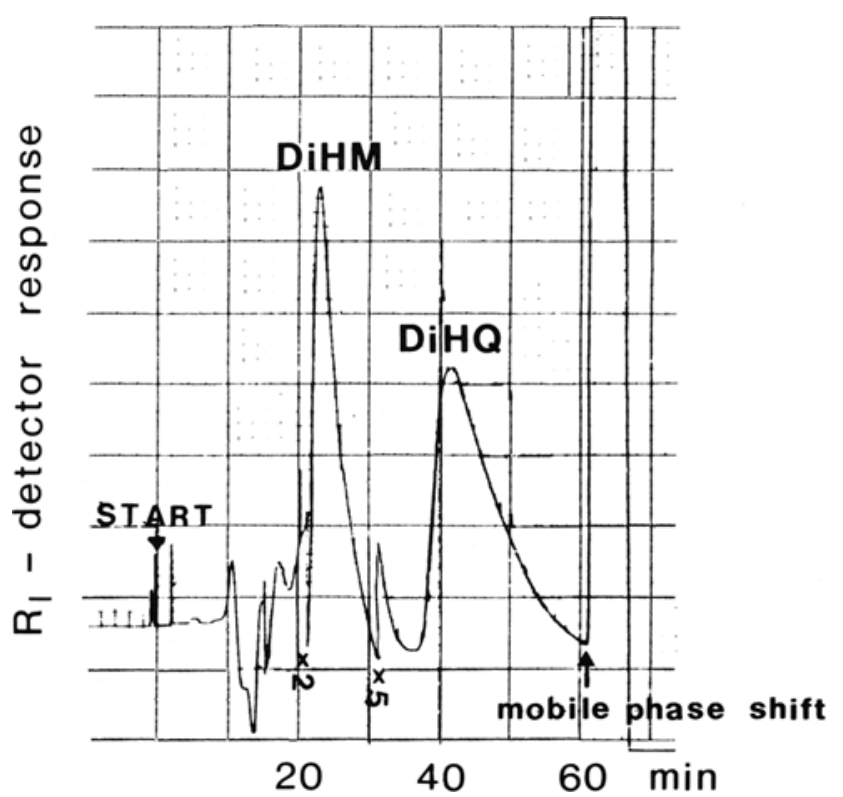

Figure 2. Preparative HPLC (2.4.2) of an ethyl acetate extract of bark (Sample 2).

has been analyzed (Sample 4) we do not know whether this is due to changes in the bark on the dead trees or reflects true differences among individual trees. The use of bark from dead trees (which had been lying for a couple of years) is, however, advantageous because of the lower content of resinous material, compared to fresh bark. Thus, the crude extract from fresh bark (Sample 4) could not be suspended in aqueous methanol, which is used for HPLC samples.

\subsection{General characteristics of dihydro- flavonols}

The nearly colourless, crystalline samples of DiHM and DiHQ obtained from preparative HPLC as described above were homogeneous according to analytical HPLC and NMR, but MS showed small amounts of impurities that were not removed by repeated recrystallizations from water.

Both compounds were optically active. DiHM and DiHQ showed $[\alpha]_{D}^{20}(c=1$, acetonewater $1: 1)$ values of $+45.2^{\circ}\left(+41.7^{\circ}(10)\right)$, and $+44.5^{\circ}\left(46^{\circ}(16)\right)$, respectively. The uncorrected melting points $\left({ }^{\circ} \mathrm{C}\right)$ were $245(225-227(10))$, and 239 (240-2 (16)), respectively.

Elementary analyses of samples that had been dried in high vacuum for $24 \mathrm{~h}$ at room temperature, indicated both compounds to be hemihydrates, as previously established in the case of DiHM (10).

The dihydroflavonols were found to be sensitive towards oxidation. Thus, HPLC analysis of an old extract that had not been protected against air and light showed the presence of transformation products giving peaks immediately after the main peaks. When the solution of DiHM and DiHQ prepared for HPLC was kept for 7 days open to air and daylight at room temperature and rerun these peaks had increased 4-5 folds.

Optically active dihydroflavonols are considered to racemize very readily by the action of acid or alkali (17). We found that DiHM was stable, i.e. no loss of optical rotation, for $24 \mathrm{~h}$ at $20^{\circ} \mathrm{C}$ when dissolved in a 1:0.4 mixture of $4 \mathrm{M}$ hydrochloric acid and methanol. When heated to $80{ }^{\circ} \mathrm{C}$ the solution lost $20 \%$ of its rotatory power during $5 \mathrm{~h}$. 
H. OUTtRup et al.: Dihydromyricetin and dihydroquercetin
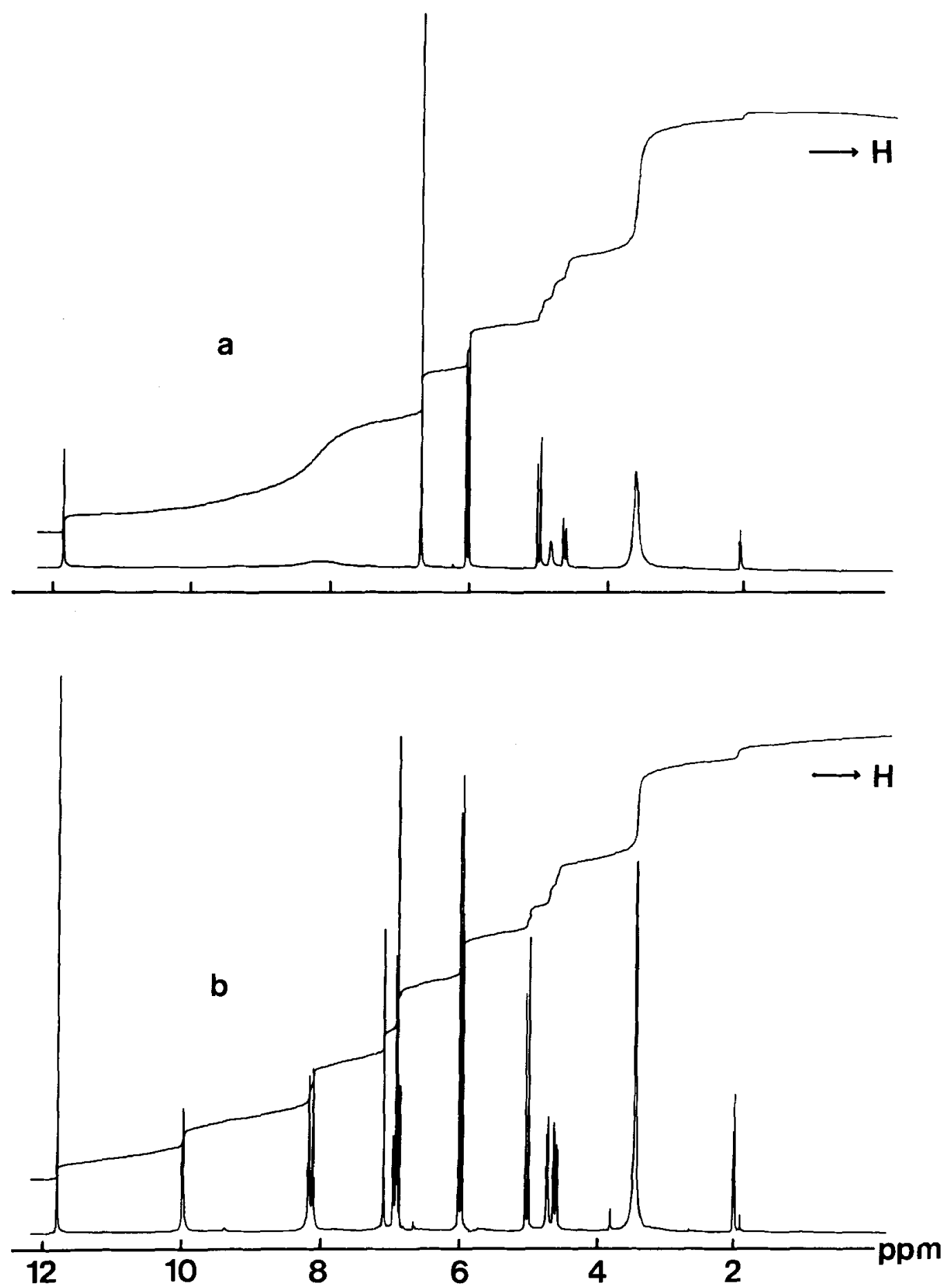

Figure 3. a: 'H-NMR spectrum of DiHM in $\mathrm{CDCl}_{3} / \mathrm{DMSO}^{-\mathrm{d}_{6}} 3: 1$.

b: 'H-NMR spectrum of DiHQ in $\mathrm{CDCl}_{3} / \mathrm{DMSO}^{-\mathrm{d}_{6}}$ 3:1. 
Table II Coupling constants $(\mathrm{Hz})$ of DiHM and DiHQ and their perdeuteroacetylated derivatives.

\begin{tabular}{|c|c|c|c|c|c|c|c|c|c|c|}
\hline \multirow{3}{*}{ Coupling } & \multicolumn{10}{|c|}{ Compound } \\
\hline & \multicolumn{3}{|c|}{ DiHM } & \multicolumn{3}{|c|}{$\begin{array}{c}\text { DiHM } \\
\text { hexaacetate } \\
-\left({ }^{2} \mathrm{H}_{3}\right)\end{array}$} & \multicolumn{2}{|c|}{ DiHQ } & \multicolumn{2}{|c|}{$\begin{array}{c}\text { DiHQ } \\
\text { pentaacetate } \\
-\left({ }^{2} \mathbf{H}_{3}\right)\end{array}$} \\
\hline & $\mathrm{a}, \mathrm{c}$ & $\mathrm{b}, \mathrm{c}$ & $\mathrm{e}$ & $\mathrm{a}, \mathrm{d}$ & $\mathrm{b}, \mathrm{c}$ & d & $\mathrm{a}, \mathrm{c}$ & c & $a, d$ & $\mathrm{~d}$ \\
\hline $\mathrm{J}(2,3)$ & 11 & 12 & 11,4 & 11 & 12 & 12,2 & 11.0 & 11.3 & 11 & 12,5 \\
\hline $\mathrm{J}(6,8)$ & - & - & 2,0 & 2 & 2 & 2,2 & - & 2,0 & - & 2,2 \\
\hline $\mathrm{J}(10,14)$ & - & - & - & - & - & - & - & 2,0 & - & 2,1 \\
\hline $\mathrm{J}(13,14)$ & - & - & - & - & - & - & - & 8,2 & - & 8,5 \\
\hline $\mathrm{J}(3, \mathrm{OH}(3))$ & - & - & 4,1 & - & - & - & - & 3,7 & - & - \\
\hline
\end{tabular}

a: Ref. (1), b: Ref. (13), c: $\left(\mathrm{CD}_{3}\right)_{2} \mathrm{CO}$-solution, d: $\mathrm{CDCl}_{3}$-solution.

e: $\mathrm{CDCl}_{3} / \mathrm{DMSO}_{6}$ 3:1-solution.

\subsection{NMR spectra}

The ${ }^{1} \mathrm{H}$ NMR spectra of DiHM and DiHQ are shown in Figure 3. The literature data on NMR of DiHM and DiHQ are scarse and we have consequently compiled our own data on these compounds and their perdeuteroacetyl deriva- tives in Table II and III $\left({ }^{1} \mathrm{H}\right)$ and IV $\left({ }^{13} \mathrm{C}\right)$ including literature data when available. The numbering of the carbon atoms is the one used earlier (14). The remaining carbons neighbouring $C(4)$ and $C(8)$ are labelled $C\left(4_{\alpha}\right)$ and $C\left(8_{\alpha}\right)$, respectively (Table IV).

Table III Chemical shifts (ppm) of DiHM and DiHQ and their perdeuteroacetylated derivatives.

\begin{tabular}{|c|c|c|c|c|c|c|c|c|c|c|}
\hline \multirow{3}{*}{$\begin{array}{l}\text { Proton } \\
\text { donor }\end{array}$} & \multicolumn{10}{|c|}{ Compound } \\
\hline & \multicolumn{3}{|c|}{ DiHM } & \multicolumn{3}{|c|}{$\begin{array}{c}\text { DiHM } \\
\text { hexaacetate } \\
-\left({ }^{2} \mathrm{H}_{3}\right)\end{array}$} & \multicolumn{2}{|c|}{ DiHQ } & \multicolumn{2}{|c|}{$\begin{array}{c}\text { DiHQ } \\
\text { pentaacetate } \\
-\left({ }^{2} \mathrm{H}_{3}\right)\end{array}$} \\
\hline & $a, c$ & b.c & e & $a, d$ & $\mathrm{~b}, \mathrm{c}$ & $\mathrm{d}$ & $a, c$ & c & $\mathrm{a}, \mathrm{d}$ & $\mathrm{d}$ \\
\hline $\mathrm{H}$ ( 2) & 4.89 & 4.88 & 4.85 & 5.44 & 5.75 & 5.43 & 4.93 & 5.00 & - & 5.43 \\
\hline$H(3)$ & 4.43 & 4.47 & 4.45 & 5.65 & 5.59 & 5.60 & 4.47 & 4.61 & 5.69 & 5.66 \\
\hline$H(6)$ & 5.88 & 5.90 & 5.93 & 6.84 & 6.63 & 6.61 & 5.89 & 5.95 & 6.38 & 6.61 \\
\hline $\mathrm{H}(8)$ & - & - & 5.98 & 6.65 & 6.83 & 6.80 & 5.89 & 5.99 & 6.72 & 6.79 \\
\hline $\mathrm{H} \quad(10)$ & 6.55 & 6.60 & 6.58 & 7.39 & 7.40 & 7.25 & 6.82 & 7.07 & 7.25 & 7.29 \\
\hline$H$ (13) & - & - & - & - & - & - & 6.82 & 6.85 & $*$ & 7.26 \\
\hline $\mathrm{H}$ (14) & 6.55 & 6.60 & 6.58 & 7.39 & 7.40 & 7.25 & 7.00 & 6.90 & 7.43 & 7.38 \\
\hline $\mathrm{OH}(3)$ & - & - & 4.94 & - & - & - & - & 4.73 & - & - \\
\hline $\mathrm{OH}(5)$ & - & - & 11.65 & - & - & - & - & 11.69 & - & - \\
\hline $\mathrm{OH}(7)$ & - & - & 10.33 & - & - & - & - & 9.91 & - & - \\
\hline $\mathrm{OH}(11)$ & - & - & 8.24 & - & - & - & - & 8.57 & - & - \\
\hline $\mathrm{OH}(12)$ & - & - & 7.54 & - & - & - & - & - & - & - \\
\hline $\mathrm{OH}(13)$ & - & - & 8.24 & - & - & - & - & 8.13 & - & - \\
\hline
\end{tabular}

* aromatic protons $(\mathrm{H}(10)-\mathrm{H}(14)$ are reported as a group in the interval 7.25-7.43 ppm. For further explanation, see notes to Table II. 


\subsection{1. 'H NMR}

In DiHM and DiHQ the assignment of $\mathrm{H}(2)$ and $\mathrm{H}(3)$ is facilitated by the observation of the ${ }^{3} \mathrm{~J}$ coupling between the $\mathrm{OH}$ proton and $\mathrm{H}(3)$. The assignment agrees with previously published data quoted in the Table II.

In the assignment of $H(10), H(13)$ and $H(14)$ in DiHQ (Table III) the literature data are wrong presumably because the closely spaced signals were not resolved at the lower field strength used earlier. A similar situation occurs for $\mathrm{H}(6)$ and $\mathrm{H}(8)$ where individual signals have not been observed in previous studies of the two molecules. The increased resolution also permits the determination of coupling constants as reported. The hydroxyl groups are observed as individual signais in the spectra even when a small amount of water is present. The aliphatic $\mathrm{OH}$-signal in both compounds is observed at approximately $4.8 \mathrm{ppm}$. The OH-signal at 11.6 $\mathrm{ppm}$ is due to the strongly chelated $\mathrm{OH}$-group in position 5. In the IR spectrum of DiHQ the observation of a $\mathrm{C}=\mathrm{O}$ absorption at $1630 \mathrm{~cm}^{-1}$ is consistent with strong chelating (1). The remaining $\mathrm{OH}$-signals are due to other phenolic groups. The assignments are based on signal intensity and consistency between the assignments in the two molecules. A slow exchange of protons between the water and non-chelated $\mathrm{OH}$-groups have been detected observing the saturation transfer from water to these groups. In the peracetylated compounds our results on DiHM are basically in accordance with the work of Miller et al. (13) but in our work all resonances have been observed and assigned.

\subsection{2. ${ }^{13} \mathrm{C} \mathrm{NMR}$}

In Table IV data for DiHQ and DiHM are listed. Proton coupled spectra yield one bond as well as a number of long range coupling constants. The assignment of $\mathrm{C}(6)$ and $\mathrm{C}(8)$ has previously (1) been based on the observation of a long range coupling to $C(6)$ and absence of long range coupling to $C(8)$. From Table IV it can be seen that both carbons display long range coupling. $\mathrm{C}(8)$ has one ${ }^{3} \mathbf{J}$ coupling to $\mathrm{H}(6)$ whereas $\mathrm{C}(6)$ has a ${ }^{3} \mathrm{~J}$ coupling to $\mathrm{H}(8)$ and a ${ }^{5} \mathbf{J}$ coupling to $H(3)$. The latter coupling is enhanced by the almost coplanar $W$ arrangement of the path between the interacting nuclei. This arrangement is also responsible for the ${ }^{3} \mathrm{~J}$ coupling from $H(3)$ to $C(4)$ which is of the same

Table IV Assignment of carbonatoms in DiHM and DiHQ and their perdeuteroacetylated derivatives.

\begin{tabular}{|c|c|c|c|c|c|c|c|c|c|}
\hline \multirow{2}{*}{$\begin{array}{l}\text { Carbon } \\
\text { No }\end{array}$} & \multicolumn{3}{|c|}{ DiHM } & \multirow{2}{*}{$\begin{array}{l}\text { DiHM } \\
\text { hexaacetate } \\
-\left({ }^{2} \mathrm{H}_{3}\right) \\
\delta \text { ppm }\end{array}$} & \multicolumn{4}{|c|}{ DiHQ } & \multirow{2}{*}{$\begin{array}{l}\text { DiHQ } \\
\text { pentaacetate } \\
-\left({ }^{2} \mathrm{H}_{3}\right) \\
\delta \text { ppm }\end{array}$} \\
\hline & $\delta \mathrm{ppm}$ & 'J Hz & ${ }^{n} \mathrm{~J} \mathrm{~Hz}$ & & $\delta p p m$ & $\delta$ ppm & ${ }^{\mathrm{I}} \mathbf{J} \mathrm{Hz}$ & ${ }^{n} \mathbf{J} \mathbf{H z}$ & \\
\hline$C(2)$ & 83.94 & 145 & $5.0 ; 5.0$ & 80.04 & & 83.78 & 146.0 & $4.0 ; 4.0 ; 4.0$ & 80.40 \\
\hline$C(3)$ & 72.44 & 142.1 & - & 73.18 & & 72.45 & 141.5 & 2.4 & 73.30 \\
\hline$C(4)$ & 197.32 & - & broad & 185.12 & & 197.44 & & & 185.30 \\
\hline$C(5)$ & $16 \grave{3.32}$ & & & 157.0 & & 163.41 & & & 156.9 \\
\hline$C(6)$ & 96.36 & 162.5 & $4.5 ; 7.0$ & 111.8 & $97.03^{a}$ & 96.41 & 162.5 & $5.0 ; 7.0$ & 111.7 \\
\hline$C(7)$ & 164.20 & - & $3.5 ; 3.5$ & 151.8 & & 164.24 & & & 151.7 \\
\hline$C(8)$ & 95.34 & 163.0 & 4.5 & 109.2 & $95.60^{\circ}$ & 95.37 & 163.0 & 4.2 & 109.2 \\
\hline$C(9)$ & 128.26 & - & broad & 134.1 & & 129.00 & - & $\Sigma \mathbf{J}-8$ & 134.4 \\
\hline$C(10)$ & 107.35 & 158.2 & $4.5 ; 7.5$ & 120.31 & & 115.18 & 159.5 & $4.5 ; 7.5$ & 125.8 \\
\hline$C(11)$ & 145.62 & - & & 144.0 & & 145.88 & - & & 143.5 \\
\hline$C(12)$ & 133.53 & - & & n.o. & & 145.06 & - & & 142.7 \\
\hline$C(13)$ & 145.62 & - & & 144.0 & & 115.18 & - & & 123.4 \\
\hline$C(14)$ & 107.35 & 158.2 & $4.5 ; 7.5$ & 120.31 & & 120.15 & 159.5 & $4.5 ; 7.5$ & 124.0 \\
\hline$C\left(4_{\alpha}\right)$ & 100.78 & - & $5.0 ; 5.0 ; 5.0$ & n.o. & & 100.81 & - & $5.0 ; 5.0 ; 5.0$ & 110.9 \\
\hline $\mathrm{C}\left(8_{\alpha}\right)$ & 167.22 & - & & 162.5 & & 167.21 & & & 162.66 \\
\hline
\end{tabular}

${ }^{a}$ Ref. (1), n.o.: not observed 


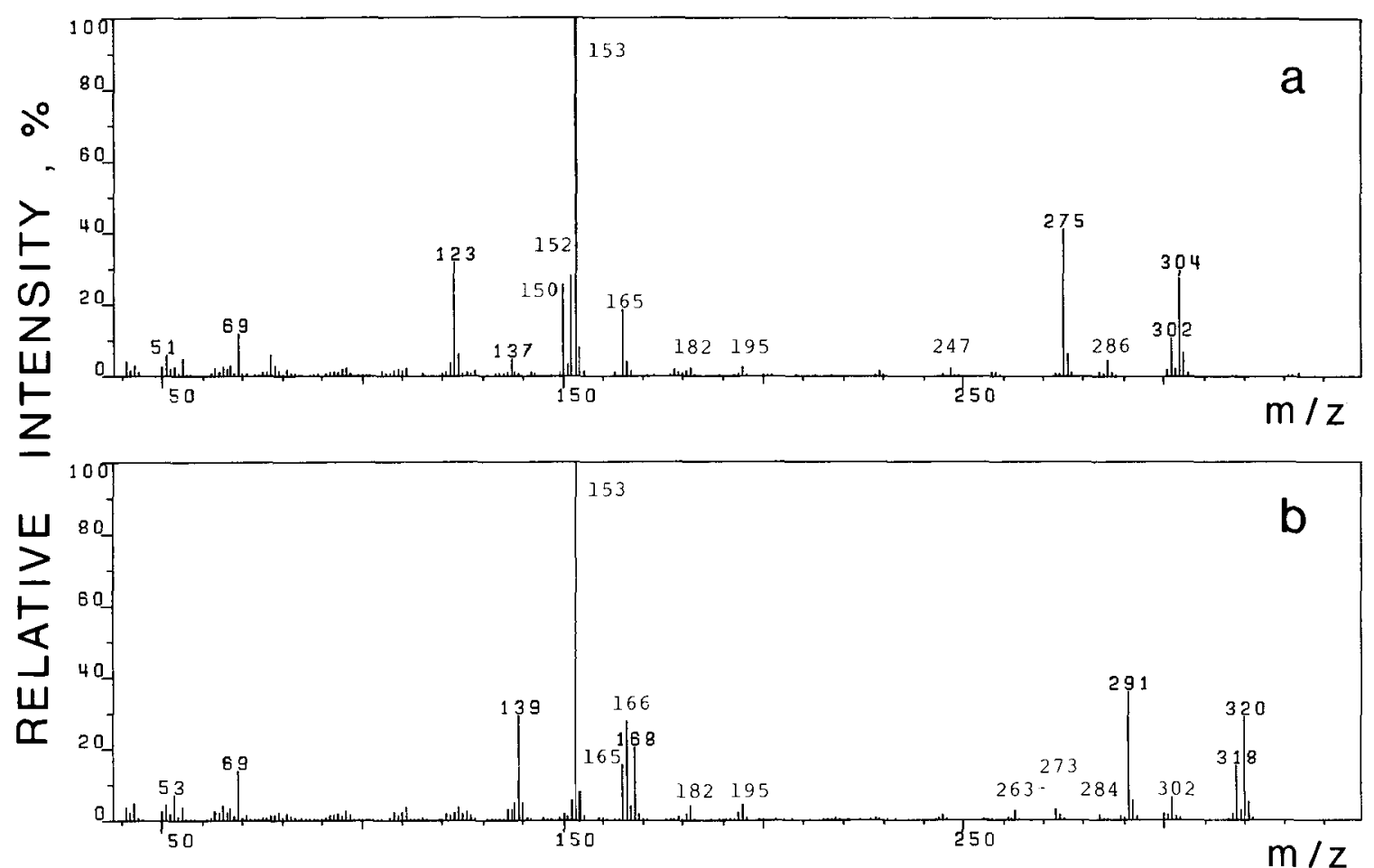

Figure 4. Mass spectra of a: DiHQ; b: DiHM.

magnitude as the ${ }^{3} \mathrm{~J}$ couplings from $\mathrm{H}(8)$ and $H(6)$. With a space filling model of DiHQ and DiHM it is realized that the phenyl group attached to $\mathrm{C}(2)$ is forced to be oriented with its ring plane perpendicular to the plane defined by the fused ring system. This configuration favours the ${ }^{4} \mathrm{~J}$ coupling from the ring protons $\mathrm{H}(10)$ and $\mathrm{H}(14)$ to $\mathrm{C}(2)$. In DiHM C(2) appears as a $1: 2: 1$ triplet while it is a $1: 3: 3: 1$ quartet in DiHQ. At the same time $C(3)$ displays a long range coupling in DiHQ while coupling is absent in DiHM. These data are consistent with a coupling in both molecules between $\mathrm{C}(2)$ and $\mathrm{H}(10), \mathrm{H}(14)$ whereas the ${ }^{2} \mathrm{~J}$ couplings between positions 2 and 3 are reduced below detection in DiHM.

\subsection{Mass spectra}

Electron impact mass spectra of flavonoid compounds, including quercetin and DiHQ, but not myricetin and DiHM, have been discussed by AUDIER (2). With our instrument, using a quartz tube to hold the sample in the direct insertion probe, we obtained spectra of quercetin and DiHQ, that are nearly identical to those reported by AUDIER. Different samples of DiHQ, however, invariably gave a small "M-2" peak at $\mathrm{m} / \mathrm{z} 302$ (Figure 4a), corresponding to the molecular ion peak of the flavonol (quercetin). This phenomenon is more pronounced in the case of DiHM. Mass spectra of this compound (Figure $4 \mathrm{~b}$ ) exhibited peaks at $\mathrm{m} / \mathrm{z} 318$ (“M-2") and 320 (M) of approximately equal intensities. During the evaporation of a sample the relative intensity of the "M-2" peak increased slowly, and the mass spectra obtained from the very last part of the sample were identical to the mass spectrum of myricetin. Since our samples of dihydroflavonols did not contain the corresponding flavonols, according to HPLC and NMR analyses, the appearance of "M-2" peaks must, at least to some extent, be caused by formation of the flavonols by 
dehydrogenation reactions. The dihydroflavonols are rather involatile, and ion source and probe had to be heated to about $200{ }^{\circ} \mathrm{C}$ in order to get spectra of reasonable intensities. The "M-2" peak intensities were reduced when the samples were placed on the edge of the quartz tube, nearest to the electron beam, and when new tubes were used. Since the spectra of the flavonols show molecular ion peaks, that are predominant base peaks, whereas the molecular ions of the dihydroflavonols give peaks of only medium intensities, the extent of dehydrogenation is much less than the ratio of "M-2" to $M$ peak intensities. The mass spectrum of DiHM is analogous with that of DiHQ (Figure 4): a number of peaks have been shifted 16 mass units upwards, whereas other peaks, including the base peak at $\mathrm{m} / \mathrm{z} 153$, are retained. Obviously, unshifted peaks correspond to ions devoid of the $B$ ring. The intensity patterns of the spectra are also very similar. Therefore, except for the "M-2" peak anomaly discussed above and the possible presence of small peaks arising from impurities, the spectrum in Figure $4 \mathrm{~b}$ is considered to be representative for DiHM. Published mass spectral characteristics of $\operatorname{DiHM}(1,7)$ are more or less at variance with our spectrum.

\section{ACKNOWLEDGEMENTS}

We are indebted to forester U. GITZ-JOHANSEN for collecting the bark samples, to Dr. I. FEILBERG, the Hørsholm Arboretum (Royal Veterinary and Agricultural University) for assistance with dendrological problems, to $\mathrm{U}$. MAXMILLING and J. WINTERBERG for technical assistance, to Dr. JACK JOHANSEN, Carlbiotech for kind help in performing preparative HPLC and to E. Knuth-Winterfeldt's Fond for financial support. The NMR equipment used was put to our disposal by the Danish Science Research Counsil (SNF).

\section{REFERENCES}

1. Agrawal. P. K., S. K. Agrawal \& R. P. Rastogi: Dihydroflavonols from Cedrus Deodara. Phytochemistry $19,893-896$ (1980)
2. Audier, H.: Étude des Composés flavoniques par Spectrométrie de Masse. Bull. Soc. Chim. France, 2892-2899 (1966)

3. Clark-Lewis, J. W.: Rev. Pure. Appl. Chem 12, 96 (1962)

4. Delcour, J. A.. D. Ferreira \& D. G. Roux: Synthesis of condensed tannins. Part 9. The condensation sequence of leucocyanidin with $(+)$-catechin and with the resultant procyanidins. J. Chem. Soc., Perkin I, 1711-1717 (1983)

5. EASTMOND, R.: The separation and identification of a dimer of catechin occurring in beer. J. Inst. Brew. 80, 188-192 (1974)

6. Fonknechten, G., M. Moll, D. Cagniant, G. KIRSH \& J. F. MULLER: Synthesis and characterisation of dimers of catechin and epicatechin. J. Inst. Brew. 89, 424-431 (1983)

7. Gellert. M. K. Szendrei \& J. Reisch: Dihydromyricetin 3-O-rhamnoside from leaves of Catha edulis. Phytochemistry 20, 1759-1760 (1981)

8. Hergert, H. L: Economic importance of flavonoid compounds: Wood and bark. In: The Chemistry of Flavonoid Compounds. T. A. Geissman ed., Pergamon Press (1962)

9. Hergert, H. L.: The flavonoids of lodgepole pine bark, J. Org. Chem. 21, 534-537 (1956)

10. HÄNSEl. R. \& J. KlafFENBACH: Optisch aktives Dihydromyricetin aus Erythrophleum africanum. Arch. Pharm. 66, 158-172 (1961)

11. JeR UMANIS. J.: Separation et identification de flavanoides par Chromatographie Liquide a Haute Performance (HPLC). Proceedings of the European Brewery Convention Congress, Berlin 1979, 309-329

12. KotAKe. M. \& T. Kubota: Über Inhaltsstoffe von Ampelopsis meliaefolia Kudo (Haku-Tya). Ann. 544, 253-271 (1940)

13. Miller, J.M.\&B.A. BoHM: Flavonoids of Leptarrhena pyrolifolia. Phytochemistry 18, 1412-1413 (1979)

14. OUtTRUP, H. \& K.SChaumburG: Structure elucidation of some proanthocyanidins in barley by 'H 270 NMR spectroscopy. Carlsberg Res. Commun. 46, 43-52 (1981)

15. Outtrup, H.: Structure of prodelphinidins in barley. Proceedings of the European Brewery Convention Congress, Copenhagen 1981, 323-333

16. PEW, J. C: A flavonone from Douglas Fir heartwood. J. Am. Chem. Soc. 70, 3031-3033 (1948)

17. Whalley, W. B.: The stereochemistry of flavonoid compounds. In: The Chemistry of Flavonoid Compounds. T. A. Geissman ed., Pergamon Press (1962) 